\title{
Digitale Ortsbrustbilder mittels Kamerabefahrung eines TBM-Bohrkopfs
}

\author{
Robert Wenighofer und Robert Galler
}

Lehrstuhl für Subsurface Engineering, Montanuniversität Leoben, Leoben, Österreich

Eingegangen 30. Oktober 2017; angenommen 6. November 2017; online publiziert 22. November 2017

Zusammenfassung: Dieser Beitrag stellt ein Kamerasystem für die geologische Dokumentation im maschinellen Tunnelbau vor. Das Kamerasystem wird im aktuellen F\&E-Projekt TBMonitor entwickelt und auf einer TBM des Bauloses Tulfes-Pfons des Brenner-Basistunnel-Projektes getestet. Es liefert hochauflösende Bilder und eine auf Fotogrammetrie basierende vollflächige 3D-Dokumentation, indem es in Vortriebspausen in Diskenkästen im Bohrkopf montiert und die gesamte Ortsbrust bis in den Kaliberbereich befahren wird. Der Montageort bewirkt, dass der Bohrkopf zur Aufnahme der Kamera nicht angepasst werden muss. Das Design, die Komponenten, die Installation, die hochautomatisierte Prozessierung der Bilder, geologische Auswertung und die Erfahrungen eines Jahres der Nutzung in einem laufenden Tunnelbauprojekt werden vorgestellt.

Schlüsselwörter: Maschineller Vortrieb, TBM,

Fotogrammetrie, Strukturgeologie

Digital Imaging of the Tunnel Face Through the Cutter Head Using Cameras

Abstract: The paper presents a camera system for the geologic documentation of the tunnel face in mechanized tunnelling. Developed in a current R\&D project TBMonitor, it is field-tested in a hard rock TBM drive of the construction lot Tulfes-Pfons of the Brenner Base Tunnel. The camera system is mounted in disc housings and enables capturing the full tunnel face. It is used in pauses of tunnel driving operations and the cutter head does not need to be adapted, providing additional apertures to accommodate the camera. Its design, components, installation, highly automized photogrammetric workflow and geologic utilization based on its high-resolution images and 3D-documentation of the

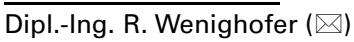

Lehrstuhl für Subsurface Engineering,

Montanuniversität Leoben,

Erzherzog-Johann Straße 3,

8700 Leoben, Österreich

robert.wenighofer@unileoben.ac.at tunnel face are presented and the experiences gained by using it in a running tunnel project over a year are discussed.

Keywords: Mechanized tunnelling, TBM, Photogrammetry, Structural Geology

\section{Einleitung}

Tunnelvortriebsmaschinen (TVMs) werden zunehmend bei Großprojekten wie dem Koralmtunnel, dem Semmeringoder dem Brenner-Basistunnel in Österreich eingesetzt, da höhere Vortriebsgeschwindigkeiten erreicht werden können. Trotz des häufigen Einsatzes von TVMs liegt der Entwicklungsstand der geologischen Dokumentation der Ortsbrust hinter jener der konventionellen Tunnelvortriebe zurück. Dies liegt vor allem an der mangelnden Einsehbarkeit der Ortsbrust. Schneidköpfe von TVMs haben nur wenige Öffnungen zur Ortsbrust, dies reduziert die Gültigkeit der geologischen Kartierung durch die Geologen.

Der Beitrag präsentiert die Ergebnisse des ersten Jahres der Verwendung eines Kamerasystems für die geologische Dokumentation der Ortsbrust im Baulos des Erkundungstunnels Tulfes-Pfons des Brenner-Basistunnel-Projektes. Das Kamerasystem wird im aktuellen F\&E-Projekt TBMonitor entwickelt. Der Tunnel wird von der ARGE TulfesPfons, einem Bieterkonsortium der STRABAG AG und Salini Impregilo S.p.A., unter Verwendung einer offenen Festgesteins-TBM mit ca. $8 \mathrm{~m}$ Durchmesser gebaut.

\section{Stand der Technik}

Im konventionellen Tunnelbau führt in der Regel der Geologe die geologische Kartierung durch. Sie wird kaum durch einen eingeschränkten Blick auf die Ortsbrust behindert. Eine Fotodokumentation kann sie vervollständigen und fortgeschrittene Werkzeuge, die auf der digitalen Bildverarbeitung basieren, können verwendet werden, um 3DDarstellungen der Ortsbrust zu erzeugen [1]. 
Im maschinellen Tunnelbau behindert der Schneidkopf jedoch die Sicht auf die Ortsbrust. Es gibt nur wenige Öffnungen durch den Schneidkopf, wie Räumer, Disken oder Mannlöcher, was die Gültigkeit der geologischen Ortsbrustkartierung einschränkt. Die Geologen nutzen diese Öffnungen, um den Zustand der Ortsbrust zu beurteilen. Die Kartierung wird nachher skizziert.

Die Situation wird durch die jüngste Entwicklung kamerabasierter Aufzeichnungssysteme verbessert. Die Auswertung der Bilder nutzt Stitchingmethoden bis hin zur Fotogrammetrie. Anfängliche Systeme, positioniert in Mannlöchern, nahmen die Bilder aus einer einzelnen Öffnung auf, während der Schneidkopf ohne Anpresskräfte gedreht wurde. Dabei waren nur schmale ringförmige Streifen der Ortsbrust erfassbar [2-4].

\section{Kamerasystem}

\subsection{Kameraeinheit}

Die Kameraeinheit wird in Vortriebspausen installiert, wo aus Diskenkästen auf unterschiedlichen Radien die Ortsbrust bei Drehung des Bohrkopfs im Leerlauf aufgenommen wird. Die Installation in den Diskenkästen ermöglicht, dass TVMs ohne zusätzliche Kameraöffnungen mit dem Kamerasystem ausgerüstet werden können, und die Anzahl der Bohrkopfdrehungen kann auf jenen Abstand eingestellt werden, den der Schneidkopf von der Ortsbrust zurückgezogen wird.

Das Kamerasystem besteht aus einer Kameraeinheit (Abb. 1a, b), die leicht und flexibel montierbar ist, und einer Steuereinheit, die über die Dauer der Aufnahme an einer Position montiert verbleibt (Abb. 1c).

Die Kameraeinheit umfasst eine Industriekamera der Allied Vision Technologies $\mathrm{GmbH}$ mit einem 2 MP Sensor und einem $5 \mathrm{~mm}$-Objektiv fester Brennweite. Auf Grund der Blendeneinstellung bieten das Objektiv und ein speziell entwickelter LED-Ring eine breite Tiefenschärfe von etwa 20 bis $200 \mathrm{~cm}$. Der LED-Ring bringt mehr als $10.000 \mathrm{~lm}$ auf, dies sorgt für eine gute Ausleuchtung von Ausbrüchen in der Ortsbrust von mehr als $1 \mathrm{~m}$ Tiefe (Abb. 2). Der LEDRing leuchtet den Kamerasichtbereich mit einer Zeitdauer von $4 \mathrm{~ms}$ aus. Diese vermeidet Bewegungsunschärfe, die die fotogrammetrische Verarbeitung der Bilder beeinträchtigt. Die Kamera ist auf eine kontinuierliche automatische Belichtung eingestellt, die die Belichtungszeit für jedes Bild aktualisiert. Es vermeidet, dass Abschnitte der Ortsbrust über- oder unterbelichtet sind. Dadurch können die Bilder gut durch eine fotogrammetrische Software verarbeitet werden.

\subsection{Steuereinheit}

Die Steuereinheit enthält einen PC, eine Energieversorgung und einen einaxialen Neigungssensor. Sie ist in einem Doppeldiskenkasten nahe dem Rotationszentrum des Schneidkopfes untergebracht. Die Kamerasteuerung, die auf dem PC läuft, löst die Kamera in einer konstanten Rate von 2 Bil-
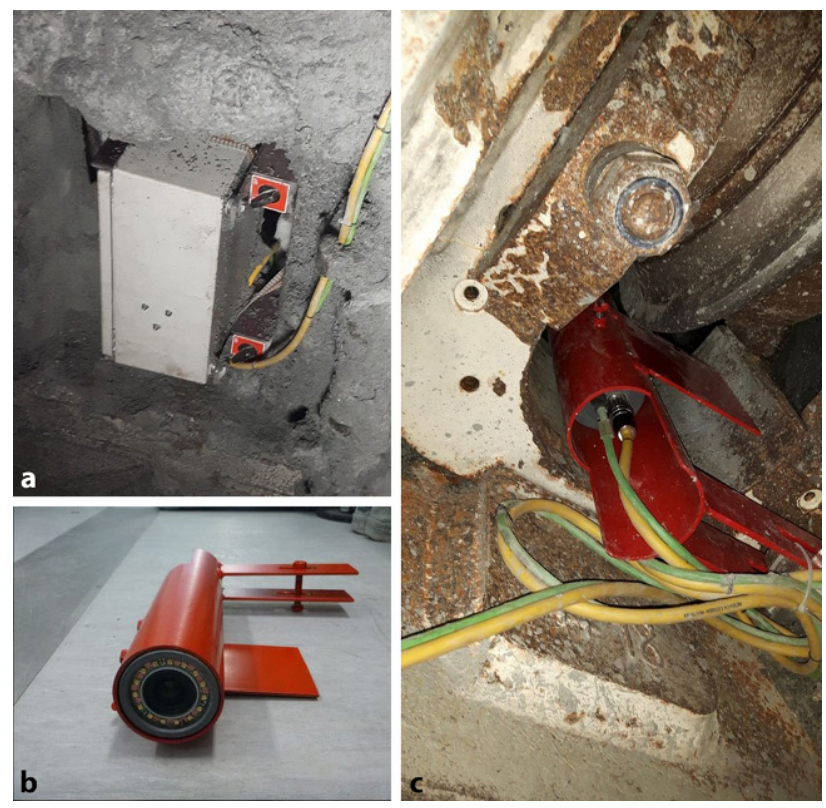

b

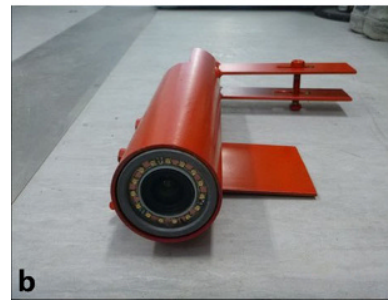

Abb. 1: a Kameraeinheit; b Kameraeinheit im Diskenkasten montiert c Steuereinheit [5]

Abb. 2: Orthofoto des vollen Querschnitts mit gleicher Beleuchtung, die Ausbrüche (Oberteil) und stabile Ortsbrustbereiche abdeckt [5]

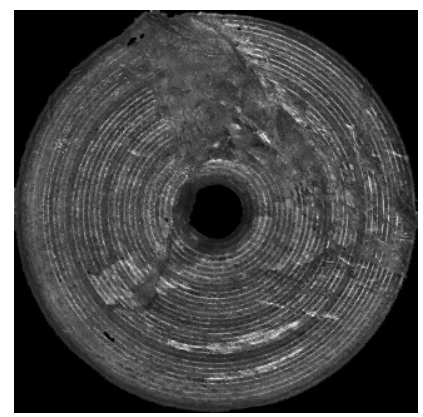

dern je Sekunde aus, was eine ausreichende Überlappung und einen Überschuss von Bildern liefert. Die Steuereinheit ist so eingestellt, dass sie automatisch die Bildaufnahme nach dem Booten des PCs startet. Jeder Beginn der Bildaufnahme beinhaltet das Erstellen eines neuen Verzeichnisses, in dem die Bilder gespeichert werden. Um die Interaktion mit der Kamerasteuerung unter den harschen Bedingungen am Schneidkopf so weit wie möglich zu vermeiden, ist keine direkte Eingabe in das System erforderlich. Der PC kann mit einem Android-Gerät (z. B. einem Smartphone) ferngesteuert werden, z. B. um die Qualität der aufgenommenen Bilder zu beurteilen. Zusätzlich bietet das Steuergerät Schnittstellen zu mehreren Kameras, die parallel betrieben werden können, um die Aufnahme zu beschleunigen.

Die Steuereinheit ist mit einem Neigungssensor ausgestattet, der ein gemeinsames Koordinatensystem für alle in einer Messkampagne aufgenommenen Bilder erstellt. Jedem Bild wird ein Winkelwert zugewiesen, der vom Neigungssensor abgerufen wird, wenn die Kamera ein Bild aufnimmt. Der Neigungswinkel und die bekannte Relativposition des Diskenkastens ermöglichen die Bestimmung der absoluten Position der Kamera im 3D-Referenzkoordinatensystem des Schneidkopfes (Abb. 3), welche wesentli- 
Abb. 3: 3D-Referenzkoordinatensystem des Schneidkop fes, blau: Disken mit der Montageposition der Kameraeinheit und Doppeldisken mit der Montageposition des Steuergerätes [5]
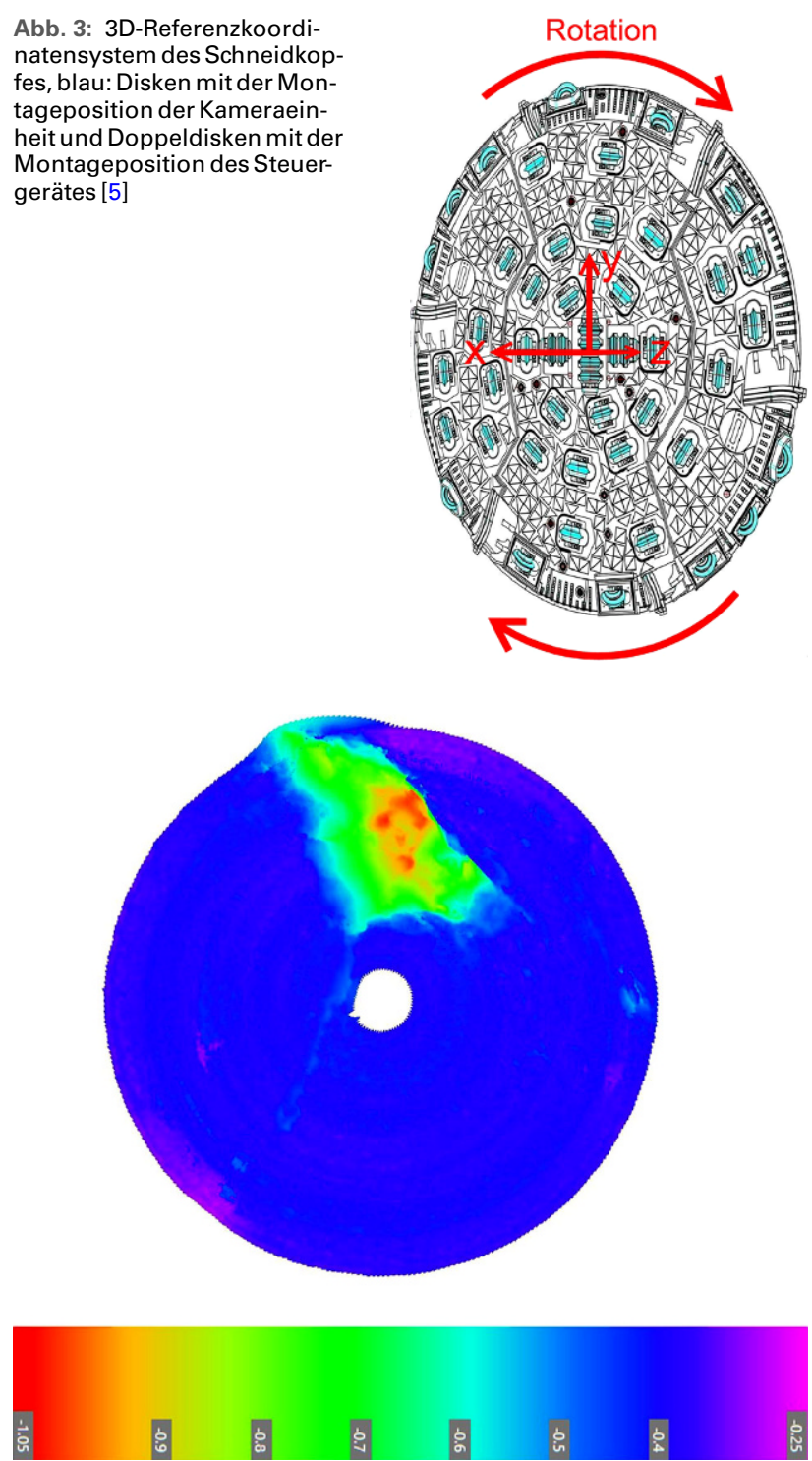

Abb. 4: Farbkodierte Reliefdarstellung der Ortsbrust von Abb. 2 und Farbskala in [m] unten [5]

che Informationen für die fotogrammetrische Verarbeitung liefert.

\subsection{Systeminstallation}

Für die Installation des Kamerasystems gibt es eine Reihe von vorbereitenden Arbeitsschritten. Nach Beendigung des TBM-Hubes muss der Schneidkopf ein paar Zentimeter zurückgezogen werden. Das Material zwischen Schneidkopf und Ortsbrust wird ausgeschuttert. Dieser Schritt wird für die Arbeitssicherheit des Bedieners durchgeführt, um ein Herunterfallen von Ausbruchsmaterial durch die Räumer zu verhindern und um eine Beschädigung des Kamerasystems auszuschließen. Die Notwendigkeit, den Schneidkopf zu waschen, hängt von der Situation der Ortsbrust ab. Wenn das ausgehobene Material feinkörnig und trocken ist, muss der Schneidkopf nur so weit mit Wasser gerei- nigt werden, dass das Staubvorkommen zwischen Kamera und Ortsbrust verringert wird. Ein Waschen kann entfallen, wenn das Aushubmaterial blockig ist.

Da nach dem Vortrieb im Bohrkopf hohe Temperaturen und Feuchtigkeit herrschen, kann das Beschlagen der vergleichsweise kühlen Kameraeinheit die Aufnahme verzögern. Um dem Problem entgegenzuwirken, kann das Kamerasystem vor dem Einbau mit Strom versorgt werden, was dazu führt, dass die Kamera eine geeignete Betriebstemperatur erreicht und im Schneidkopf schneller akklimatisiert wird.

Die Steuereinheit ist in den Gehäusen der Doppeldisken nahe dem Bohrkopfzentrum untergebracht, sodass für die Dauer der Aufzeichnung ein konstantes Referenzsystem für die Schneidkopfdrehung erhalten wird. Die Kameraeinheit kann flexibel in verschiedenen Diskenkästen montiert werden und die Anzahl der Kameramontagen kann entsprechend dem Ortsbrustabstand gesteuert werden. Für die Montage der Kamera im roten Halter (Abb. 1a) müssen die Diskenkästen zuerst ausgekratzt werden. Der Transport schwerer Hilfsmittel zur Montage oder Reinigung der Diskenkästen ist nicht notwendig. Das Anschlusskabel muss nach dem Befestigen der beiden Geräte sicher mit Kabelbinder am Schneidkopf montiert werden.

\subsection{Bildaufnahme}

Zur Bildaufnahme wird der Schneidkopf manuell etwas mehr als $360^{\circ}$ ohne Anpresskräfte gedreht. Anschließend werden die Kameras nach jeder Umdrehung herausgenommen und in weiteren Diskenkästen eingebaut. Die manuelle Drehung des Schneidkopfes dauert ca. $3 \mathrm{~min}$. Die Reihenfolge der besetzten Diskenkästen kann arbeitsergonomisch gewählt werden. Im Allgemeinen werden Diskenkästen benachbarter Räumerkammern ausgewählt. In dem gegebenen Projekt beträgt der Abstand zwischen den Disken $9 \mathrm{~cm}$. Die Anzahl der Abstände zwischen zwei Kameraspuren kann in Abhängigkeit von der Entfernung zwischen Schneidkopf und Ortsbrust gewählt werden.

Das System wird vorwiegend während der Erkundungsbohrungen, der Wartung der TBM, der Verlängerung des Förderbandes und anderen Vortriebspausen verwendet. Die Wartung des Schneidkopfs, wie z. B. das Wechseln von Disken, erlaubt kein gleichzeitiges Drehen des Schneidkopfes für Kameraaufnahmen.

\section{Fotogrammetrische Prozessierung}

Die fotogrammetrische Verarbeitung der Bilder einer vollflächigen Ortsbrustaufnahme profitiert von den aktuellen Entwicklungen im Bereich unbemannter Luftfahrzeuge (UAV). Für die Auswahl der entsprechenden Software wurden mehrere Pakete evaluiert. Auf die Fähigkeit, automatisierte Bildprozessierungen zuzulassen, wurde dabei ein besonderes Augenmerk gelegt.

Aufgrund einer umfangreichen Python-Anwendungsprogrammierschnittstelle (API) wird PhotoScan von Agisoft 


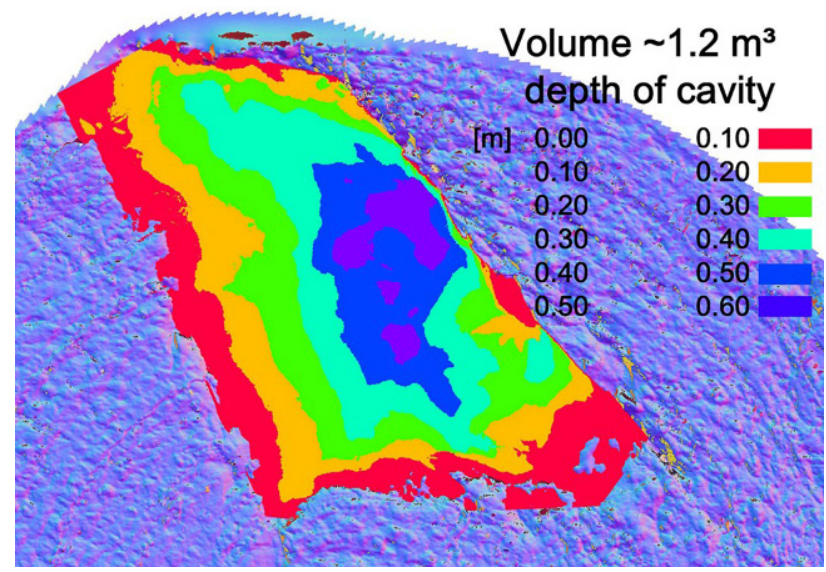

Abb. 5: Volumsbestimmung eines Ausbruchs aus der Ortsbrust von Abb. 2, die Tiefe des Ausbruchs ist entsprechend der hinzugefügten Farbskala farbcodiert [5]

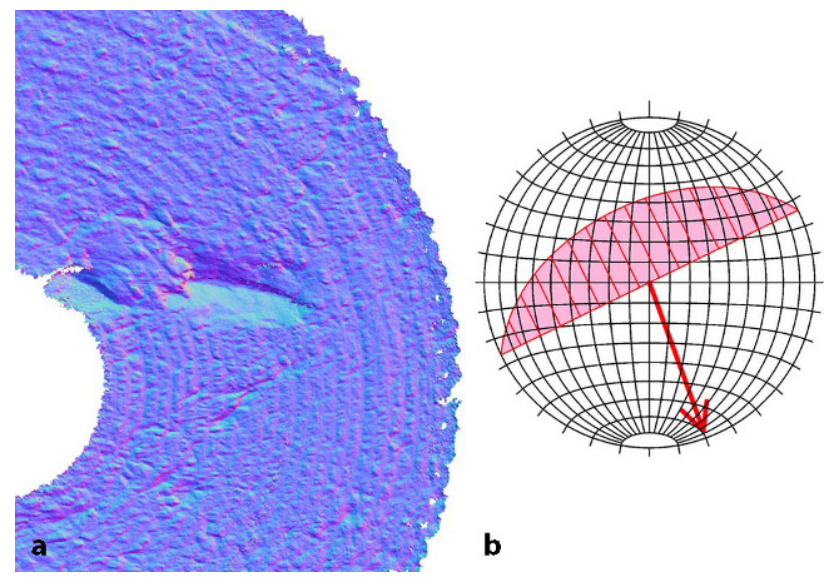

Abb. 6: Darstellung der Diskontinuität (Ausbruch) (a) im Schmidt'schen Netz (b) einschließlich der Vortriebsrichtung (roter Pfeil). Das Bild zeigt die farbkodierten Normalvektoren der Punktwolke zur besseren Erkennung von Diskontinuitäten [5]

LLC [6] verwendet. PhotoScan ist eine bekannte Lösung zur Verarbeitung von UAV-Bilddaten. Der Einsatz einer bewährten Software hat den großen Vorteil, einen hochwertigen Support zu haben und in unzähligen Projekten praxiserprobt zu sein. Mit PhotoScan können die vom Bediener manuell durchzuführenden Schritte auf die Auswahl einer Datei der Kamerakalibrierung und einer Textdatei reduziert werden, welche Orientierungsdaten (wie z. B. die Position, an der das Bild aufgenommen wurde) enthält. Orientierungsdaten sind erforderlich, um das Zusammenmatchen der großen Anzahl ( 1000) von Bildern zu beschleunigen. Die hochautomatisierte fotogrammetrische Prozessierung dauert in der Regel ca. 50 min und umfasst:

- Laden der Kamera-Kalibrierungsdateien, Bilder und Orientierungsdaten

- Zusammenmatchen der Bilder

- Berechnen der Punktwolke der gesamten Ortsbrust

- Berechnen eines rund 250 MP-Orthofotos (Abb. 2) aus der Punktwolke

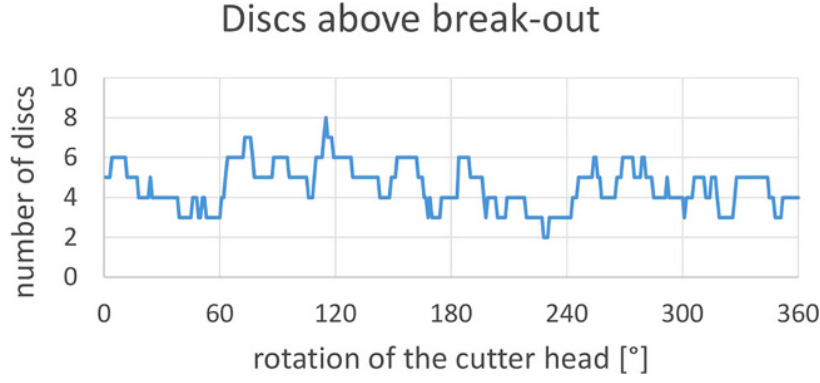

Abb. 7: Anzahl der Disken, die auf Grund von Ausbrüchen der Ortsbrust (von Abb. 2) nicht über eine volle Umdrehung des Bohrkopfes angedrückt werden

- Interpolation eines digitalen Höhenmodells (DEM) (Abb. 4) aus der Punktwolke

- Exportieren aller Ergebnisse zur weiteren Verarbeitung in das Dateisystem.

\section{Auswertung der digitalen Ortsbrustaufnahmen}

Die für die Ortsbrust charakteristischen und in der Literatur vorgeschlagenen Auswertungsmethoden reichen von der Volumenbestimmung von Ausbrüchen, wie sie von CloudCompare oder Autodesk Civil 3D (Abb. 5) unterstützt wird, bis hin zur Darstellung von Diskontinuitäten im Schmidt'schen Netz (Abb. 6).

Anhand der von Ausbrüchen eingenommenen Flächen kann die asymmetrische Belastung des Schneidkopfes untersucht werden. Da die Anordnung der Disken bekannt ist, ermöglichen großflächige Aufnahmen der Ortsbrust ein Erfassen, welche und wie viele Disken des Bohrkopfs gleichzeitig die Ortsbrust im Vortrieb nicht berühren (Abb. 7). Eine solche Rauigkeit der Ortsbrust verursacht eine abrupte Belastungsänderung und eine erhöhte Belastung der übrigen Disken, die die Lebensdauer und die Materialermüdung der Schneidwerkzeuge beeinflussen.

Die digitale Ortsbrustaufnahme führt zu einer objektiveren geologischen Kartierung, die eine unwiderlegbare Grundlage der geologischen Beurteilung für Auftraggeber und Auftragnehmer darstellt. Hochauflösende Orthofotos zeigen Details, die hinter dem Bohrkopf für den Geologen nicht sichtbar sind. Farbkodierte Reliefdarstellungen zeigen hingegen an erster Stelle Ausbrüche (Abb. 4). Reliefpräsentationen können jedoch auch ringförmige Merkmale umfassen, wie im Überlappungsbereich von Abb. 8 unten zwischen Kameraaufnahmen aus unterschiedlichen Diskenkästen zu sehen ist. Es treten blaue, vertiefte ringförmige Zonen auf, die als Zonen mit erhöhter Disken-Belastung missdeutet werden können. Wenn man ihre Position mit dem Orthofoto vergleicht, zeigt sich, dass diese Merkmale mit Abschnitten niedrigerer Beleuchtung verbunden sind. Diese Merkmale können bei Bedarf entfernt werden, indem die Kameraspuren näher zueinander angeordnet werden. Dies führt jedoch zu einer zeitaufwendigeren Bildaufnahmephase. 
Abb. 8: Geologische Kartierung mit digital aufgenommener Ortsbrust unter Verwendung eines Orthophotos (a) und einer farbcodierten Reliefdarstellung (b). sf Schieferung, $h$ Harnisch, qu Quarzgang, st Störung [5]

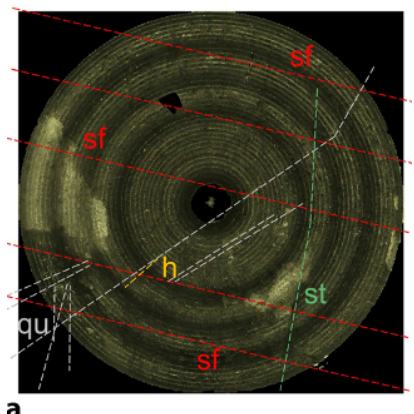

a

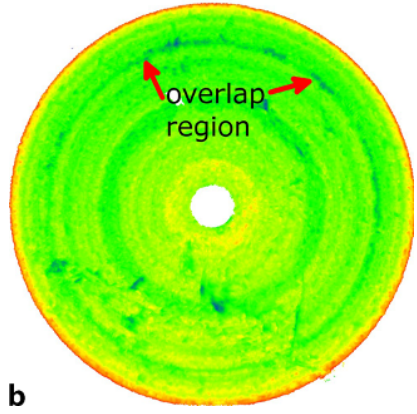

b

Eine Voraussetzung für die Aufnahme der Bilder ist jedoch ein stabiler Zustand der Ortsbrust insofern, als ein Zurückziehen des Bohrkopfes um einige Zentimeter möglich ist und der Bohrkopf betreten werden kann.

\section{Schlussfolgerungen}

Der Beitrag präsentiert ein Kamerasystem zur geologischen Dokumentation der Ortsbrust im maschinellen Tunnelbau. Das System kann sowohl mit einer als auch mit mehreren Kameras verwendet werden. Nach mehr als einem Jahr Einsatz des Kamerasystems hat sich die fotogrammetrische Verarbeitung als ein geeignetes Mittel erwiesen, um die Ortsbrust sogar in der rauen Umgebung eines TBM-Vortriebs digital darzustellen. Der Einsatz des Kamerasystems muss jedoch die Anforderungen an die Arbeitssicherheit für den Bediener erfüllen, da das Betreten des Bohrkopfs unumgänglich ist. Großflächiges, über den TBM-Querschnitt hinausgehendes Versagen der Ortsbrust kann aus Sicher- heitsgründen häufig nicht erfasst werden, da Hereinfallen von Ausbruchsmaterial durch die Räumer die Arbeitssicherheit gefährdet. Das Kamerasystem erfordert auf Grund fehlender Wartungsphasen eine gute Koordination durch den Auftragnehmer. Da das System in 17"-Diskenkästen montiert werden kann, bietet es eine große Flexibilität bei der Montage, die den Abstand zwischen Bohrkopf und Ortsbrust berücksichtigt. Das Kamerasystem stellt hochauflösende Orthofotos und eine 3D-Dokumentation der Ortsbrust bereit. Sie sind digitale, für Auftraggeber und Auftragnehmer zweifelsfreie Belege für die Ortsbrustcharakterisierung.

Danksagung. Erhebliche Teile der präsentierten Entwicklung wurden im Rahmen des nationalen Forschungsprojekts TBMonitor von der Österreichischen Forschungsförderungsgesellschaft FFG gefördert.

Funding. Open access funding provided by Montanuniversität Leoben.

Open Access Dieser Artikel wird unter der Creative Commons Namensnennung 4.0 International Lizenz (http://creativecommons.org/licenses/ by/4.0/deed.de) veröffentlicht, welche die Nutzung, Vervielfältigung, Bearbeitung, Verbreitung und Wiedergabe in jeglichem Medium und Format erlaubt, sofern Sie den/die ursprünglichen Autor(en) und die Quelle ordnungsgemäß nennen, einen Link zur Creative Commons Lizenz beifügen und angeben, ob Änderungen vorgenommen wurden.

\section{Literatur}

1. Fasching, W.; Gaich, A.; Schubert, W.: Data Aquisition in Engineering Geology: An Improvement of Acquisition Methods for Geotechnical Rock Parameters, Felsbau 19 (2001), pp 93-101

2. Henzinger, M.: Geotechnische Dokumentation der Ortsbrust eines TBM-Vortriebs mittels moderner messtechnischer Methoden, Masterarbeit, Montanuniversität Leoben, 2013

3. Schuller, E.; Galler, R.; Barwart, S.; Wenighofer, R.: The transparent face - development work to solve problems in mechanized hard rock tunnelling, Geomechanics and Tunnelling, 8 (2015), No. 3 pp 200-210

4. Gaich, A.; Pötsch, M.: 3D images for digital tunnel face documentation at TBM headings - application at Koralmtunnel lot KAT2, Geomechanics and Tunnelling, 9 (2016), No. 3, pp 210-221

5. Wenighofer, R.; Galler, R.; Chmelina, K.; Six, G.: Using Cameras in TBM Cutterheads for Geological Documentation of the Tunnel Face, Proceedings of the World Tunnel Congress 2017 of International Tunnelling and Underground Space Association ITA-AITES, Bergen, Norway, 2017, p 6

6. Agisoft LLC: PhotoScan Python Reference, Release 1.2.5, 2016 